\title{
A METHOD FOR SELECTING OPTIMAL SECTIONS FOR MEMBERS OF SKELETAL STRUCTURES
}

\author{
CHU DuC NHA \\ Ministry of Education and Training
}

\begin{abstract}
This paper presents a simple method for selecting optimal sections for members of skeletal structures from an initially given set of sections. This is an extension of evolutionary structural optimization (ESO) to the sizing optimization problem with discrete design variables. Member sensitivity index for section sizing is derived from the optimality criterion. Optimization process is an iterative process of analysis, sensitivity calculation and section selection until optimality criterion is satisfied or constraints are violated. The proposed optimization procedure has been implemented into a structural analysis and optimization program called FEMOPT written on MATLAB programming language. Illustrative examples demonstrate the effectiveness of the proposed method.
\end{abstract}

\section{Introduction}

In design of skeletal structures (trusses, frames), member sections must be selected from an initially given set of sections. This is clear for steel structures where sections have to be chosen from available range produced by manufacturers. Selecting optimal sections for members to minimize the weight (or cost) of a structure is always the desire of any designer and this can be achieved by employing a structural optimization method. However, due to complexity of structural optimization when dealing with real problems and not popularity of optimization software packages, the problem is usually solved by trying several sets of member sections and choose the best among them [1]. The obvious limitation of this "trial" method is not able to identify the optimal solution if it is not included in trial sets.

Based on idea of Evolutionary Structural Optimization (ESO) for shape and topology optimization of plates under stress consideration proposed by Y. M. Xie and G. P. Steven [2], the author has developed ESO methods for plate and truss topology optimization $[3,4,5,6]$ and for optimal design of plate with discrete thicknesses [7], where the objective is the minimum weight and constraints are imposed on displacements. Illustrative examples provided in these works show the simplicity and efficiency of the proposed methods.

This paper presents an extension of ESO methods to frame sizing optimization problem with discrete variables, i.e. member sections must be chosen from an initially given set of sections. The optimization problem can be stated as following

By selecting sections for members from a given set of sections, minimize the weight of the structure subject to constraints imposed on some displacement components 
The most difficulty in this problem is that the general member section have to be defined by several dimensions, therefore one member may relate to several design variables. This destroys separability of the problem and makes more difficulties in deriving optimality criterion. Using member weights as intermediate variables will overcome this difficulty. One member relate to only one design variable, offering more convenience in deriving optimality criterion and member sensitivity analysis.

\section{Sensitivity analysis}

\subsection{Effect of member section sizing on displacements}

Consider a skeletal structure comprising $n$ members. When the $i^{\text {th }}$ member changes its section to the next smaller or larger section, the weight and stiffness matrix of the $i^{\text {th }}$ member as well as the weight and stiffness matrix of the whole structure will take the following changes

$$
\Delta w_{i}=w_{i}^{*}-w_{i}
$$

and

$$
[\Delta K]=\left[K^{*}\right]-[K]=\left[\Delta K^{i}\right]=\left[K^{i}\right]^{*}-\left[K^{i}\right]
$$

where $w_{i},\left[K^{i}\right]$ and $[K]$ are weight, stiffness matrix of $i^{\text {th }}$ member and stiffness of the structure before sizing; $w_{i}^{*},\left[K^{i}\right]^{*}$ and $\left[K^{*}\right]$ are weight, stiffness matrix of $i^{\text {th }}$ member and stiffness of the structure after sizing, respectively.

Considering equilibrium equations before sizing

$$
[K]\{u\}=\{P\}
$$

and after sizing

$$
[K+\Delta K]\{u+\Delta u\}=\{P\} .
$$

By assuming that load vector remains unchanged $\{\Delta P\}=0$ and ignoring higher order member, the change in the displacement vector is as

$$
\{\Delta u\}=-[K]^{-1}[\Delta K]\{u\} .
$$

Introduce a virtual unit load $\left\{F_{j}\right\}$, where the $j^{\text {th }}$ component equals unity and others are set to zeros. From (2.5), the change in $u_{j}$ by changing section of the $i^{\text {th }}$ member will be

$$
\Delta u_{j}=\left\{F^{j}\right\}^{T}\{\Delta u\}=-\left\{F^{j}\right\}^{T}[K]^{-1}[\Delta K]\{u\}=-\left\{u^{j}\right\}^{T}[\Delta K]\{u\}
$$

where $\left\{u^{j}\right\}$ is called as a virtual displacement vector, which is solution of equation (2.3) for the unit load. Reminding (2.2), we have

$$
\Delta u_{j}=-\left\{u^{j}\right\}^{T}[\Delta K]\{u\}=-\left\{u^{i j}\right\}^{T}\left[\Delta K^{i}\right]\left\{u^{i}\right\}
$$


where $\left\{u^{i}\right\}$ and $\left\{u^{i j}\right\}$ are real and virtual displacement vectors of the $i^{\text {th }}$ element, which can be extracted from the real displacement vector $\{u\}$ and virtual displacement, vector $\left\{u^{j}\right\}$.

By using the unit load method, a displacement component at a given point can be calculated by formula

$$
u_{j}=\sum_{i=1}^{n} \alpha_{i j}
$$

where

$$
\alpha_{i j}=\left\{u^{i j}\right\}^{T}\left[K^{i}\right]\left\{u^{i}\right\}
$$

is the element virtual energy, which is also called as the $i^{\text {th }}$ element contribution to $u_{j}$.

Assuming the change in displacements are small, the change in element virtual energies can be approximated by the quantity

$$
\Delta \alpha_{i j}=\alpha_{i j}^{*}-\alpha_{i j}=\left\{u^{i j}\right\}^{T}\left[K^{i}\right]^{*}\left\{u^{i}\right\}-\left\{u^{i j}\right\}^{T}\left[K^{i}\right\}\left\{u^{i}\right\}=\left\{u^{i j}\right\}^{T}\left[\Delta K^{i}\right]\left\{u^{i}\right\}
$$

Comparing (2.7) and (2.10), we have

$$
\Delta u_{j}=-\left\{u^{i j}\right\}^{T}\left[\Delta K^{i}\right]\left\{u^{i}\right\}=-\Delta \alpha_{i j}
$$

Thus, the change in the specified displacement, in absolute values, is approximated by the change in the element virtual energy when the element change its section. Formula (2.11) provides an easy way to evaluate the effect of element section sizing on displacements.

It is obvious that reducing section of the element with small $\Delta \alpha_{i j}$ will make small change in the displacement. When all element can be reduced by the same amount of weight, reduction of the element with smallest value $\left|\Delta \alpha_{i j}\right|$ is the best choice, because the resulting structure will have the same weight while the displacement results in the least change. When elements change their weights differently by section reduction, the efficiency of section reduction also depends on change of element weight. Comparing two elements with the same values of $\left|\Delta \alpha_{i j}\right|$, it is obvious that section reduction of the element resulting in more weight reduction will produce a lighter weight structure with the same displacement. It is clear that section reduction of element with smaller ratio $\left|\Delta \alpha_{i j}\right| /\left|\Delta w_{i}\right|$ is more efficient. To understand the physical meaning of ratio $\left|\Delta \alpha_{i j}\right| /\left|\Delta w_{i}\right|$, consider the original optimization problem using Lagrange multiplier approach for derivation of optimality criterion.

\subsection{Member section sizing sensitivity index}

a) Single displacement constraint. Consider a problem of minimization of the structural weight

$$
W=\sum_{i=1}^{n} w_{i}
$$


subjected to a constraint imposing on a single displacement component given as

$$
\left|u_{j}\right|-u_{j}^{*} \leq 0
$$

where $u_{j}^{*}$ is the limiting value for absolute value of displacement $u_{j}$. The Lagrangian of the problem is as

$$
L=W-\lambda\left(\left|u_{j}\right|-u_{j}^{*}\right)
$$

where $\lambda$ is a Lagrange multiplier.

In general, beam section can be described by several dimensions (section depth, flange width, flange thickness, web thickness ...), which means that several design variables have to be defined for one member. To preserve separability of the problem and generality of discussion, we choose the element weights $w_{i}(i=1, n)$ as intermediate variables. Taking derivatives of Lagrangian by $w_{i}$, the optimality criterion has the following form

$$
\frac{\partial L}{\partial w_{i}}=\frac{\partial W}{\partial w_{i}}-\lambda\left|\frac{\partial u_{j}}{\partial w_{i}}\right|=1-\lambda\left|\frac{\partial u_{j}}{\partial w_{i}}\right|=0 \quad(i=1, n)
$$

This can be approximated by

$$
1-\lambda\left|\frac{\Delta u_{j}}{\Delta w_{i}}\right|=0 \quad(i=1, n)
$$

Taking into account formulas (2.1) and (2.11) which give the change in weight and displacement due to sizing element section, the optimality criterion (2.16) can be written in the following form

$$
\gamma_{i}=\left|\frac{\Delta \alpha_{i j}}{\Delta w_{i}}\right|=\frac{1}{\lambda}=\text { const } \quad(i=1, n)
$$

Equation (2.17) expresses the following optimality criterion: at the optimal solution, absolute values of ratios between the changes in the element virtual energies and the element weights are equal for all elements.

Investigations show that reducing the element with smallest $\gamma_{i}$ can create more uniform values of $\gamma_{i}$, which means the resulting structure is more close to optimal solution. Iterative process of analysis and section reduction of elements with smallest values of $\gamma_{i}$ will result in lighter weight while displacements increase slowly. This means the value $\gamma_{i}$ can characterize effectiveness of element section reduction.

Thus, the member (element) section sizing sensitivity index for a single constraint, or simply the member sensitivity index, can be defined as

$$
\gamma_{i}=\gamma_{i j}=\left|\frac{\Delta \alpha_{i j}}{\Delta w_{i}}\right|=\frac{\left|\left\{u^{i j}\right\}\left[\Delta K^{i}\right]\left\{u^{i}\right\}\right|}{\left|\Delta w_{i}\right|} \quad(i=1, n)
$$


It is noted that calculation of member sensitivity index by (2.18) is straight forward. Using a structural analysis package, by one run we can have real and virtual displacements for real and virtual unit loads. The changes in the element weight and element stiffness matrix is determined by assuming that the element section is going to change to the next smaller or larger available section in the given set.

b) Multiple displacement constraints. For the weight minimization problem subjected to $m$ displacement constraints

$$
\left|u_{j}\right|-u_{j}^{*} \leq 0 \quad(j=1, m)
$$

the Lagrangian will have the following form

$$
L=W-\sum_{j=1}^{m} \lambda_{j}\left(\left|u_{j}\right|-u_{j}^{*}\right)
$$

where $\lambda_{j}(j=1, m)$ are Lagrange multipliers. Optimality conditions are as

$$
\frac{\partial L}{\partial w_{i}}=\frac{\partial W}{\partial w_{i}}-\sum_{j=1}^{m} \lambda_{j}\left|\frac{\partial u_{j}}{\partial w_{i}}\right|=1-\sum_{j=1}^{m} \lambda_{j}\left|\frac{\partial u_{j}}{\partial w_{i}}\right|=0 \quad(i=1, n)
$$

where $\lambda_{j}>0$ for the active constraints $\left|u_{j}\right|-u_{j}^{*}=0$ and $\lambda_{j}=0$ for passive constraints $\left|u_{j}\right|-u_{j}^{*}<0$. Optimality conditions $(2.21)$ can be approximated by

$$
1-\sum_{j=1}^{m} \lambda_{j}\left|\frac{\Delta u_{j}}{\Delta w_{i}}\right|=0 \quad(i=1, n)
$$

Taking into account (2.1) and (2.11), we have

$$
1-\sum_{j=1}^{m} \lambda_{j}\left|\frac{\Delta \alpha_{i j}}{\Delta w_{i}}\right|=0 \quad(i=1, n)
$$

or

$$
\eta_{i}=\sum_{j=12}^{m} \lambda_{j}\left|\frac{\Delta \alpha_{i j}}{\Delta w_{i}}\right|=1 \quad(i=1, n)
$$

Formula (2.24) expresses an optimality criterion for multiple displacement constraints as follows: at the optimal solution, the weighted sum of ratios between absolute changes in the element virtual strain energy and the element weight are equal to unity for all elements, where weighting parameters are Lagrange multipliers. Investigations show that reducing section of the element with the smallest value $\eta_{i}$ will create a more uniform state of values $\eta_{i}$, producing a design closer to the optimal solution. 
Thus, the member (element) section sizing sensitivity index for multiple constraints, or simply the member sensitivity index, can be defined as

$$
\eta_{i}=\sum_{j=1}^{m} \lambda_{j}\left|\frac{\Delta \alpha_{i j}}{\Delta w_{i}}\right|=\sum_{j=1}^{m} \lambda_{j} \gamma_{i j} \quad(j=1, n)
$$

where $\gamma_{i j}$ are defined by (2.18), which means that the member sensitivity index for multiple displacement constraints is the weighted sum of the member sensitivity indexes for each constraint, where weighting parameters are Lagrange multipliers.

\subsection{Determination of Lagrange multipliers}

The Lagrange multipliers can be determined by the iteration formula, the ratio formula or linear equations as discussed in [5]. The simplest way is to use the ratio formula

$$
\cdot \lambda_{j}=\left(\frac{\left|u_{j}\right|}{\varphi u_{j}^{*}}\right)^{1 / b} \quad(j=1, m)
$$

where $b$ is a step size, $\varphi$ is a constraint limit scaling parameter, defined by

$$
\varphi=\max _{j=1, m} \varphi_{j}=\max _{j=1, m}\left(\frac{\left|u_{j}\right|}{u_{j}^{*}}\right)
$$

which is used to scale all constraint limits to make the most potential active constraint become the active constraint. The use of constraint limit scaling will keep member sections (design variables) within the given set of sections (discrete values).

\section{Evolutionary optimization procedure}

The evolutionary optimization procedure for weight minimization subject to displacement constraints is as follows:

Step 1. Choose the largest available sections for elements in the finite element model. Step 2. Analyse for real loads and virtual unit loads corresponding to constraints. Step 3. If any of constraints is violated, go to Step 7. Otherwise, go to Step 4.

Step 4. Calculate sensitivity indexes $\gamma_{i j}$ for all elements for every constraint. For multiple constraints, scale constraint limits, determine Lagrange multipliers and calculate sensitivity indexes $\eta_{i}$ for all elements.

Step 5. If all element sensitivity indexes are equal, i.e. optimality criterion is satisfied, go to Step 7. Otherwise, go to Step 6.

Step 6. Reduce sections of a number of elements which have smallest sensitivity indexes and repeat Step 2 to Step 6.

Step 7. Stop. Calculate effective weight for all obtained designs. Optimal solution is the one that has minimum effective weight.

It is obvious, the proposed optimization procedure is an iterative process of three main steps: analysis, sensitivity calctlation and section reduction. Initially, all 
elements are assigned the largest available sections. After analysis, if constraints are violated, the process is terminated because it is not possible to obtain feasible designs by reducing member section. Further during optimization process, the members reached the smallest section are kept unchanged.

Sensitivity indexes are calculated by assuming member sections change to the next smaller sections. When all sensitivity indexes are equal, i.e. the optimality criterion is satisfied, the optimal solution is reached. In other cases, the optimal solution is the one that has minimum effective weight and it may not be the last design just before the constraints are violated. The effective weight is the weight of the structure when the most active constraint reaches its limit. In the case of trusses, the effective weight is equal to the actual weight multiplying by limit scaling parameter $\varphi$ defined by (2.27).

In the proposed procedure, the number of elements to be reduced their sections at each step should be identified. This can be done by one of the following ways:

(1) by a number of elements;

(2) by ratio (percentage) of the number of elements to be reduced at each step to total number of elements, which is called element changing ratio (ECR);

(3) by material (weight) ratio to be removed from structure at each step, which is called material removal ratio (MRR).

It should be noted that derivation of element sensitivity indexes are based on assumption that the change in displacements at each iteration are small. Therefore, we should choose ECR or MRR small enough to ensure gradual change in stiffness, so that displacements can change slowly. It is clear that the changes in displacements are not only depend on the number of elements to be reduced their sections, but also the rate of the change (how much the change) in section dimensions, which are called step size. For bars in trusses, the step size is a decrement of section area. In the case of beams in frames, where sections are defined by several dimensions, the decrement of member weight can be used as a common step size. When the step size increases, the amount of material to be removed from the structure increases, which makes larger changes in displacements even when the number of elements to be reduced their sections is unchanged. In this case, smaller ECR should be provided. It is observed that MRR closer relates to the change rate of displacements, so that MRR should be used for control the change rate of displacements between to successive iterations. In the author's former work concerning plate optimization with discrete thicknesses [6], the values MRR $=1 \%$ was used giving adequate results, so this value can also used for optimization of frames and trusses.

\section{Computer implementation: Frame analysis and optimization pro- gram FEMOPT}

A computer program for frame analysis and optimization, called FEMOPT (Finite Element Method and Optimization), has been developed for implementing the proposed evolutionary optimization procedure [8]. FEMOPT is built by using main 
program with exchangeable subroutines (modules) approach and written by MATLAB programming tools with open source codes, which offers more flexibility for further development.

FEMOPT (version 1.0) has two functions: static analysis and optimization. Static analysis function gives nodal displacements, support reactions and member end forces. Optimization function offers effective way for selecting optimal member sections from initially given sets of discrete sections. After each iteration, section distribution, structure's actual weight, values of constrained displacements, maximum and minimum sensitivity indexes are kept in files. These data allow us to calculate the effective weight of obtained designs and identify the optimal design with the lightest effective weight.

FEMOPT can analyse and optimize any arbitrary skeletal structure (plane or space frames, trusses) under multiple static loading conditions. Loads can be point forces or distributed loads. Boundary conditions may be given as fixed (zero) or prescribed (non-zero) displacements. Results can be output to files in numerical or in graphical format.

Structural data (nodal coordinates, loads, boundary conditions, member connection, material and section properties) are provided in the matrix format (MATLAB file) or the text formatted file (FEMOPT data file) similar to STRAND6 or SAP90 data files. FEMOPT can even run on STRAND6 or SAP90 text formatted data file. This is very useful for comparing FEMOPT with STRAND6 or SAP90. Data for controlling optimization process (maximum number of iterations, number of virtual unit load cases, number of sizing elements / ECR / MRR, section group identifications) are given in a separate file. In general, each member or each group of members can have its own set of allowable sections (section group). The initially given set of sections (comprising all section groups), virtual unit load cases corresponding to displacement constraints are input additionally into the structural data file.

\section{Examples}

\subsection{Optimization of ten-bar truss}

Consider a ten bar truss given in Fig. 1 with dimension $\ell=2 \mathrm{~m}$, material properties $E=2.1 \times 10^{11} \mathrm{~N} / \mathrm{m}^{2}, \rho=7800 \mathrm{~kg} / \mathrm{m}^{3}$ and point load $P=0.5 \times 10^{6} \mathrm{~N}$. Member section areas can be assigned any value from the set $A=\{10,15,20,25,30\}\left(\mathrm{cm}^{2}\right)$. The problem is to seek optimal section area distribution so that the weight is minimized while keeping the absolute value of vertical displacement at loaded node 6 less than $5 \mathrm{~cm}$. This is a typical example that was used by many researchers to examine optimization procedures $[9,10]$.

The problem has one constraint under one load case. Besides the real load, we have to input a virtual unit load at node 6 . The five different section areas are input in five different bar section properties in ascending order with identification numbers ranging from 1 to 5 . Initially, all bars are assigned the largest section area $A=30 \mathrm{~cm}^{2}$ with section identification number 5 . Using optimization program 
FEMOPT with 4 bars having section areas reduced to the next lower values at each step. The optimization process is terminated after 12 iteration when all bars have the smallest section while the specified displacement is still less than the limit. Table 1 gives the change in member section identification numbers.

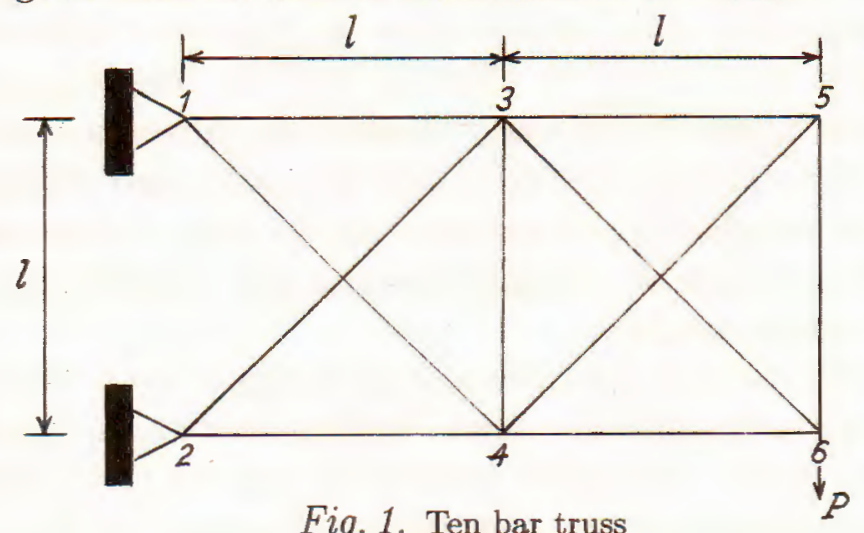

Fig. 1. Ten bar truss

Table 1. Member section identification numbers during optimization process

\begin{tabular}{|c|c|c|c|c|c|c|c|c|c|c|c|c|c|}
\hline \multirow{3}{*}{ Bar } & \multirow{2}{*}{$\begin{array}{l}\text { Position } \\
\left(\mathrm{N}_{i}-\mathrm{N}_{j}\right)\end{array}$} & \multicolumn{12}{|c|}{ Member section identification numbers at each iteration } \\
\hline & & 1 & 2 & 3 & 4 & 5 & 6 & 7 & 8 & 9 & 10 & 11 & 12 \\
\hline & & - & - & - & - & - & - & - & - & - & - & - & - \\
\hline 1 & $1-3$ & 5 & 5 & 5 & 5 & 5 & 5 & 5 & 5 & 4 & 3 & 2 & 1 \\
\hline 2 & $3-5$ & 5 & 4 & 3 & 2 & 1 & 1 & 1 & 1 & 1 & 1 & 1 & 1 \\
\hline 3 & $2-4$ & 5 & 5 & 5 & 5 & 5 & 5 & 5 & 5 & 4 & 3 & 2 & 1 \\
\hline 4 & $4-6$ & 5 & 4 & 4 & 4 & 4 & 3 & 3 & 2 & 1 & 1 & 1 & 1 \\
\hline 5 & $3-4$ & 5 & 4 & 3 & 2 & 1 & 1 & 1 & 1 & 1 & 1 & 1 & 1 \\
\hline 6 & $5-6$ & 5 & 4 & 3 & 2 & 1 & 1 & 1 & 1 & 1 & 1 & 1 & 1 \\
\hline 7 & $1-4$ & 5 & 5 & 5 & 5 & 4 & 3 & 2 & 1 & 1 & 1 & 1 & 1 \\
\hline 8 & $2-3$ & 5 & 5 & 5 & 5 & 5 & 4 & 3 & 2 & 2 & 1 & 1 & 1 \\
\hline 9 & $3-6$ & 5 & 5 & 5 & 5 & 5 & 5 & 4 & 3 & 2 & 1 & 1 & 1 \\
\hline 10 & $4-5$ & 5 & 5 & 4 & 3 & 3 & 2 & 1 & 1 & 1 & 1 & 1 & 1 \\
\hline
\end{tabular}

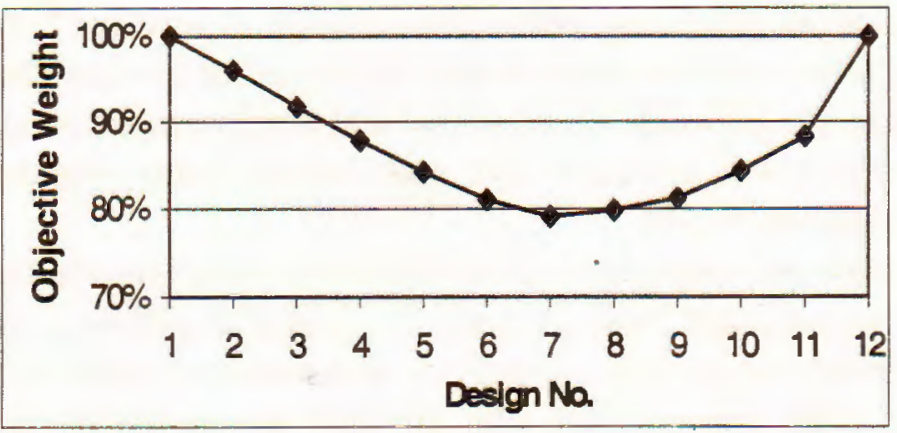

Fig. 2. Effective weight during optimization process 


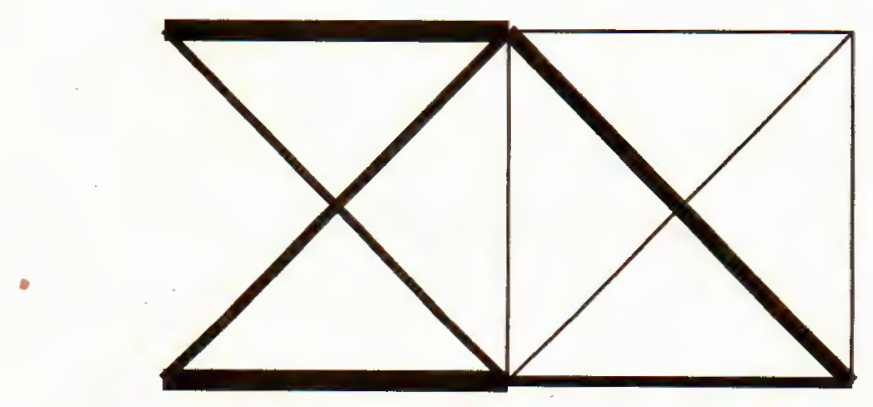

Fig. 3. Optimal section distribution

To identify the optimal solution, we should calculate the effective weight of the truss at each step by scaling all section areas by a factor so that the vertical displacement at node 6 is at the limit value $5 \mathrm{~cm}$. For trusses, the scaling parameter (factor) is equal to the ratio between the current and the limit values of constrained displacement. Fig. 2 shows the change in the effective weight during optimization process, where the minimum is reached at step 7 , which is $79 \%$ of the initial effective weight. Thus, the optimal solution is the section distribution at step 7 as shown in Fig. 3, where thicker lines describe larger sections.

For this example, using proposed evolutionary optimization procedure implemented in Program FEMOPT, we can obtain the optimal solution with the material saving of $21 \%$ of initial weight. It should be noted that the uniform section distributions with largest (at step 1) and smallest area (at step 12) are equivalent in terms of material utilization, but not the optimum. This means that the relative section distribution over the whole structure is more important than their absolute values. Therefore, where is appropriate, we can scale the all member sections by a factor to satisfy other constraints on stresses, member stability, ... without destroying its optimality.

\subsection{Optimization of an arch bridge}

The problem is to seek an optimum design of an arch with given span and height. The design load are three point vertical loads $P=10 \mathrm{kN}$ acting simultaneously at points A, B and C. Young's modulus $E=2 \times 10^{11} \mathrm{~Pa}\left(\mathrm{~N} / \mathrm{m}^{2}\right), \rho=7800 \mathrm{~kg} / \mathrm{m}^{3}$. Section areas are selected from the initial given set

$$
A=0.1 ; 0.2 ; 0.3 ; 0.4 ; 0.5 ; 0.6 ; 0.7 ; 0.8 ; 0.9 ; 1.0\left(\mathrm{~cm}^{2}\right) .
$$

The limit of $20 \mathrm{~mm}$ is imposed on vertical displacements at the loaded point. This optimization problem has three constraints under one load case.

In this problem, a designer has large freedom to choose shape (nodal grid), topology (member connection), and member sections. To solve shape optimization problem is much more difficult because the nodes coordinates are changing and is not discussed here. Evolutionary method for truss topology optimization starting from an initially chosen ground structure has been given in works $[5,6]$, by which the optimal topology with uniform section areas for this arch bridge is found as 
shown in Fig. 4.

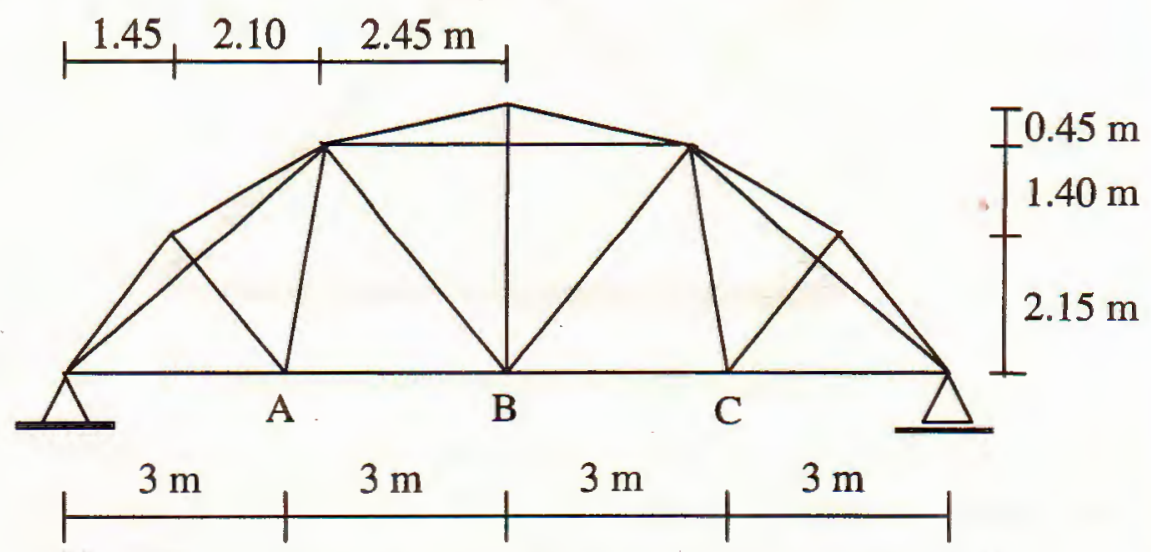

Fig. 4. Optimal topology for the arch bridge $[5,6]$

Starting from this optimal topology, we try to seek optimal section distribution among members by using our proposed method. Ten sections with ten given areas are input in ascending order with section identification numbers ranging from 1 to 10. Initially all members are assigned section identification No. 10 with the largest area. The displacement limit is set at $50 \mathrm{~mm}$. Using FEMOPT with 4 members being reduced section to the next smaller sections, which equivalent to removing about 2 to $2.5 \%$ material from the structure. The change in the effective weight is given in Fig. 5. The minimum effective weight is reached at step 31, which is equal $81,5 \%$ of the initial value. Fig. 6 shows the optimal section distribution for the arch bridge where the thicker lines describe the larger sections. Members in symmetrical places are changing similarly during the optimization process.

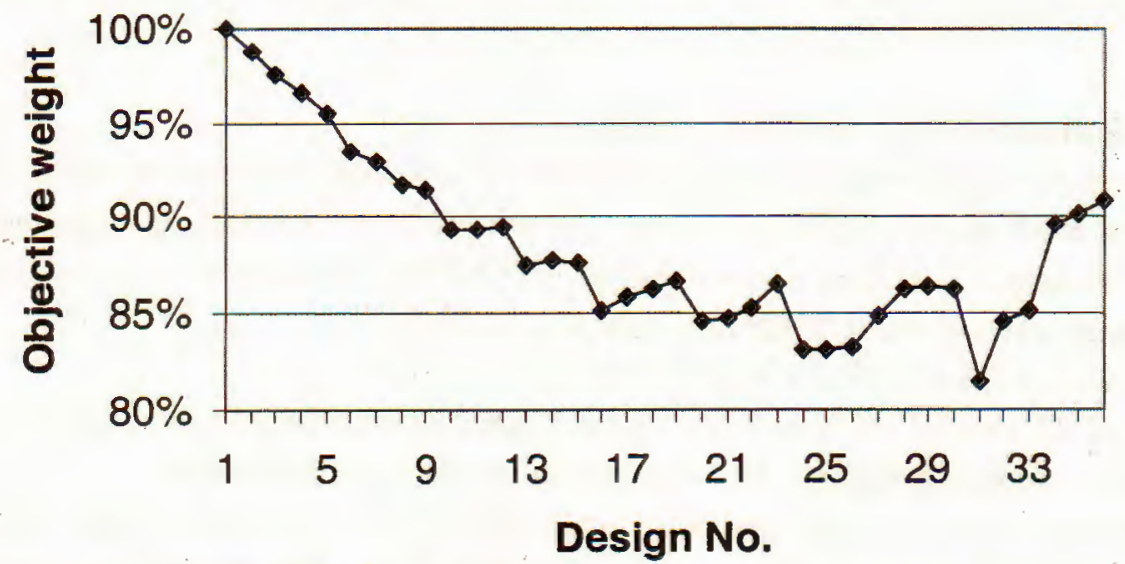

Fig. 5. Effective weight during optimization process

During optimization process, although the effective weight increase at some step, in general it decreases to the minimum value and than starts to increase. If the process is continued, sooner or later, all members will reach to the smallest section. With the material removal rate of 2 to $2.5 \%$ at each step, we can arrive to the 
uniform section distribution with the smallest section at some step between 36 and 45.

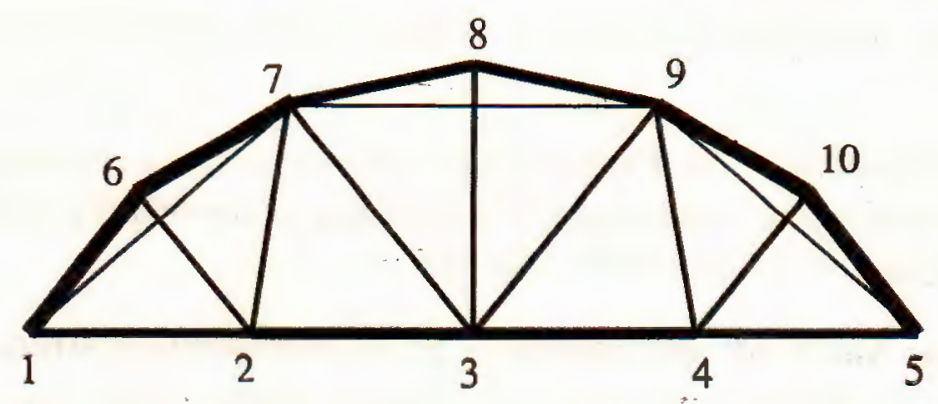

Fig.6. Optimal section distribution

\section{Conclusion}

This paper has presented a theoretical basics of an evolutionary optimization method for selecting optimal member sections for skeletal structures from an initially given set of sections. Provided examples show the effectiveness of the proposed method. Optimization program FEMOPT can serve as a tool for optimal section design of frames or trusses. FEMOPT developed on MATLAB environment with open source codes is suitable for research and training purposes in the field of structural analysis and design.

This work was a result of Research Project B99-34-52 supported by Ministry of Education and Training and was improved by the support of Council for Natural Sciences of Vietnam.

\section{REFERENCES}

1. Do Duc Thang. Some issues on rational connection and geometry of space frame systems for wide span roofs and industrial workshops, Proceedings of the 6th National Congress on Mechanics, Hanoi, 3-5 Dec. 1997, 231-237 (in Vietnamese).

2. Xie Y. M. and Steven G. P. A simple evolutionary procedure for structural optimization, Computers \& Structures, Vol. 49, 1993, 885-896.

3. Chu Duc Nha, Xie Y. M., Hira A. and Steven G. P. An evolutionary procedure for structural optimization with displacement constraints, in Building for the 21st Century - Proceedings of the Fifth East Asia - Pacific Conference on Structural Engineering and Construction, Vol. 2, 1995, 1091-1096.

4. Chu Duc Nha, Xie Y. M., Hira A. and Steven G. P. Evolutionary structural optimization for problems with stiffness constraints, Finite Elements in Analysis and Design, Vol. 21, 1996, 239-251. 
5. Chu Duc Nha. Evolutionary structural optimization method for systems with stiffness and displacement constraints, Ph.D. Thesis, 1997, Victoria University of Technology, Melbourne, Australia. (Call No, at Vietnam National Library: L6608).

6. Chu Duc Nha and Xie Y. M. Evolutionary method for truss topology optimization subject to displacement constraints, Proceedings of the 6th National Congress on Mechanics, Hanoi, 3-5 Dec. 1997, 185-191.

7. Chu Duc Nha, Xie Y. M. and Steven G. P. An evolutionary structural optimization method for sizing problems with discrete design variables, Computers \& Structures, Vol. 68, 1998, 419-431.

8. Chu Duc Nha. Structural analysis and optimization program FEMOPT, Selection of scientific research studies, University of Civil Engineering, No. 3, 2000, 71-79 (in Vietnamese).

9. Haftka R. T., Gurdal Z. and Kamat M. P. Elements of Structural Optimization, 2nd revised edition, 1990, Kluwer Academic Publishers, Dordrecht.

10. Morris A. J. (ed.). Foundation of Structural Optimization: A Unified Approach, 1982, John Wiley \& Sons, New York.

Received July 3, 2001

\section{VỀ MỘT PHUONG PHÁP LỰA CHOQN TIẾT DIỆN TỐI UU \\ CHO CÁC PHẦN TƯ TRONG KẾT CẤU HỆ THANH}

Bài báo giới thiệu một phương pháp đơn giản cho phép lựa chọn tiết diện tối ưu cho các phần tư của kết cấu hệ thanh từ tập hợp tiết diện cho trước. Đây là sự mở rộng của phương pháp tối ưu tiến hóa cho bài toán tối ưu tiết diện ngang với biến thiết kế rời rạc. Chỉ số độ nhạy dùng để thay đổi tiết diện phần tư được thiết lập từ tiêu chuẩn tối ưu. Thuật giải tối ưu là quá trình lặp lại các bước phân tích kết cấu, tính độ nhạy phần tử và thay đổi tiết diện cho đến khi tiêu chuẩn tởi ưu được thỏa mãn hay các điều kiện ràng buộc bị vi phạm. Thuật giải tối ưu được cụ thể hóa thành Chương trình tính toán và tối ưu kết cấu $\mathrm{FEMOPT}$ viết bằng công cụ lập trình MATLAB. Các ví dụ minh họa cho thấy hiệu quả của phương pháp đề xuất. 